\title{
Buffer Layer Assisted Growth of Ag Nanoparticles in Titania Thin Films
}

\author{
L. Zilberberg, S. Mitlin, H. Shankar and M. Asscher
}

Institute of Chemistry, Edmund J. Safra Campus,

The Hebrew University of Jerusalem, Jerusalem 91904, Israel

\section{Supporting Information:}

The examination of UV-VIS spectra of a number of pristine amorphous, invacuum annealed amorphous, in-air annealed nanocrystalline $\mathrm{TiO}_{\mathrm{x}}$ films on a sapphire substrate reveals discernible and systematic changes in the spectral range $200-800 \mathrm{~nm}$ which covers the fundamental absorption below $360 \mathrm{~nm}$, the absorption in the visible range by various color centers and the absorption tail stretched over a large part of the visible range in the bathochromic direction and associated with an absorption band(s) in the infra-red region and the light absorption by quasi-free electrons. ${ }^{1}$

\section{The optical absorption in the pristine RLAD nanoscale $\mathrm{TiO}_{\mathrm{x}}$ films.}

The absorption coefficient, $\alpha$, is defined conventionally as

$$
\alpha=\frac{2.303 \cdot A}{d}=\frac{2.303 \cdot \log \left(I_{0} / I\right)}{d}
$$

where $A$ is the absorbance, $d$ - the thickness of the film, $I_{0}$ and $I-$ the intensities of the incident and transmitted fluxes, respectively. For the direct interband transition, the quantity $(\alpha h v)^{2}$ increases with the photon energy above the absorption edge as follows: ${ }^{1,2}$

$$
(\alpha h v)^{2}=\text { Const }_{D} \cdot\left(h v-E_{\text {Opt.Edge }}\right)
$$

where $E_{\text {Opt.Edge }}$ is the optical edge for the direct transition, and $h v$ is the photon energy. On the other hand, for the indirect transitions in amorphous semiconductors the following dependence has been proposed following Tauc's studies: ${ }^{1,3,4}$

$$
\sqrt{\alpha h v}=\text { Const }_{I n} \cdot\left(h v-E_{\text {Opt.Edge }}\right)
$$

The onset of the optical absorption in this spectral range is defined as the intercept of the linear extrapolation of the steep part of an absorption curve and a base line $^{4,5}$ as shown in Figure $\mathrm{S} 1$ for the amorphous $\mathrm{TiO}_{\mathrm{x}}$ film obtained by 40 cycles of 
the RLAD deposition with a thickness of $18 \mathrm{~nm}$. At the initial stage of exposure to ambient atmospheric conditions, this nanoscale amorphous film is characterized by a relatively steep absorption curve with the onset at $321 \mathrm{~nm}(3.86 \mathrm{eV})$. The comparison with reported values for the onset of optical absorption of anatase at $385 \mathrm{~nm}(3.22 \mathrm{eV})$ and rutile at $415 \mathrm{~nm}(2.99 \mathrm{eV})^{5}$ indicates on the significantly larger optical gap in the present nanoscale amorphous $\mathrm{TiO}_{\mathrm{x}}$ films relative to the bulk crystalline phases.

Prepared by the hydrolysis of $\mathrm{TiCl}_{4}$ in a water-in-oil microemulsion, $\mathrm{TiO}_{2}$ nanoparticles, with the estimated size of $15 \AA$ or $12 \AA$ while assumed to be crystallized in anatase or rutile forms, respectively, have been found to exhibit the onset of the optical absorption at $345 \mathrm{~nm}(3.6 \mathrm{eV})^{4}$ which is again at a lower energy relative to the present values. These characteristic differences can be attributed, first of all, to the disordered and to a certain degree nonstoichiometric structure of asgrown $\mathrm{RLAD} \mathrm{TiO}_{\mathrm{x}}(\mathrm{OH})_{\mathrm{z}}$ films. Signature of the exciton confinement effect is not evident or, perhaps, overwhelmed by more significant factors in these amorphous films. It is found that a $\mathrm{TiO}_{\mathrm{x}}$ film prepared by $3 \mathrm{RLAD}$ cycles with a roomtemperature annealing step after each deposition cycle exhibits the onset of the optical absorption at $337 \mathrm{~nm}(3.68 \mathrm{eV})$ and the optical gap obtained in the direct coordinates at $3.72 \mathrm{eV}$ (Table S1, entry 2). These are bathochromically shifted with respect to 321 $\mathrm{nm}(3.86 \mathrm{eV})$ and the direct gap at $4.13 \mathrm{eV}$ for the film prepared by 40 RLAD cycles (Table S1, entry 1). Moreover, as also evident in Figure S1, the exposure of the 40cycle $\mathrm{TiO}_{\mathrm{x}}$ film to an ambient atmosphere for 5 hours leads to a discernible bathochromic shift from 321 to $331 \mathrm{~nm}$ in the onset and the increase in the slope of the absorption curve near the optical edge. Together with the increase in the value of the absorption coefficient this indicates on a relatively slow, steady process of structure ordering by a continuing polycondensation of Ti oxy-hydroxides species with an incomplete coordination within the $\mathrm{TiO}_{\mathrm{x}}$ matrix at a room temperature. In contrast, the 3-cycle $\mathrm{TiO}_{\mathrm{x}}$ films appears to be optically stable under ambient conditions implying that the room-temperature in-vacuum annealing of each deposited titania layer leads to a titania configuration with a lower number of defects.

Another subtle but, nonetheless, important spectral feature in Figure S1 resides in the decrease in an absorption tail, which extends in the bathochromic direction over almost an entire visible range, upon the atmospheric exposure. Considered in combination with the increase in the fundamental absorption and the bathochromic 
shift of the latter, it indicates evidently on the depopulation of the conduction band due to the oxidation of $\left\{\mathrm{Ti}_{\mathrm{i}}{ }^{*}, \mathrm{Ti}_{\mathrm{i}}{ }^{\cdots}\right\}$ interstitials in accord with the following reaction.

$$
\mathrm{O}_{2(\text { gas })}+\left\{\mathrm{Ti}_{\mathrm{i}}^{\bullet \bullet}, \mathrm{Ti}_{\mathrm{i}}^{\bullet \bullet \bullet}\right\}^{\text {in-air anneling }} \rightarrow \mathrm{Ti}_{\mathrm{Ti}}^{\mathrm{X}}+2 \mathrm{O}_{\mathrm{O}}^{\mathrm{X}}+(2,3) h^{\bullet}
$$

This oxidation process upon the exposure to an ambient atmosphere is reckoned also for ordering and the crystallization of amorphous titania.

Comparatively large optical gaps in the present titania films and the bathochromic shift in the onset of fundamental absorption upon an atmospheric annealing can be attributed to the Burstein-Moss effect. ${ }^{1}$ Indeed, if as-grown titania films contain a significant number of Ti interstitials, which act as donors in populating the conduction band to an appreciable depth, then the absorption edge for the given films is hypsochromically shifted with respect to more ordered materials with the lower concentration of donors and the higher density of states at the top of the valence band and at the bottom of the conduction band. ${ }^{1}$

Evident in Figure S2, the analysis of spectral profiles in respective coordinates proposed for the direct and indirect transitions ${ }^{2,3,5}$ yields values of $4.13 \mathrm{eV}$ and 4.01 $\mathrm{eV}$ for the direct allowed optical transition in the initial moment and after 5 hours into the exposure, respectively, which could be compared with values of $4.04 \mathrm{eV}$ and 4.03 $\mathrm{eV}$ for $\mathrm{TiO}_{2}$ nanoparticles, comprising 70-80\% of an amorphous phase and 20-30\% of the anatase phase, with the diameter of $21 \AA$ and $133 \AA$ (or $267 \AA$ ), respectively. ${ }^{5}$ For fully crystallized rutile 220 -nm thick films there have been reported values of 3.44$3.51 \mathrm{eV}$ for the direct transitions ${ }^{3,5}$ (i.e. significantly red-shifted relative to the present values) whereas for amorphous and poorly crystallized films the indirect transitions have been found at 3.32-3.35 $\mathrm{eV}^{6}$ These values can be compared with our results for the indirect transition at 3.41 and $3.38 \mathrm{eV}$ for the $18 \mathrm{~nm}$ thick $\mathrm{TiO}_{\mathrm{x}}$ film at the initial moment and after 5 hours under atmospheric conditions, respectively, as shown in Figure S2. The present nanocrystalline rutile film, the Raman and XRD spectra of which are shown in Figure 4B in the main manuscript, is characterized by values of $3.74 \mathrm{eV}$ and $3.36 \mathrm{eV}$ for the direct and indirect optical transitions, respectively, and the Scherrer crystalline size of $10 \mathrm{~nm}$. The direct transition gap at $3.74 \mathrm{eV}$ is still significantly higher than $3.44-3.51 \mathrm{eV}$ for the fully crystalline thick rutile film. The present nanocrystalline anatase film, the Raman and XRD spectra of which are shown in Figure 4A in the main manuscript is characterized by values of $3.79 \mathrm{eV}$ and $3.59 \mathrm{eV}$ for the direct and indirect optical transitions, respectively, and the Scherrer 
crystalline size of $50 \mathrm{~nm}$. The optical gaps appear to be comparable with those of the present rutile film as shown in Table $\mathrm{S} 1$ and significantly lower than corresponding values for the amorphous titania.

UV-VIS spectra of as-grown amorphous RLAD titania film at 40 cycles (18$\mathrm{nm}$ thick) and the nanocrystalline rutile and anatase films are shown in Figure S5. Evidently, the nanocrystalline films are characterized by the substantially stronger absorption in the fundamental and by the corresponding bathochromic shift in the absorption edge in comparison with the amorphous titania film. This is the manifestation of the crystallization process accompanied with the oxidation of $\mathrm{Ti}_{i}{ }^{n}$. species present in the as-grown matrixes. It is noteworthy that the UV-vis spectra of as-grown and in-vacuum annealed to $775 \mathrm{~K}$ films are identical which imply evidently that the in-vacuum crystallization process is inhibited by the interstitial titanium species and the sapphire substrate. In contrast, we have found that in-air annealing procedures lead to the crystalline titania phases in particular because of the oxidation of the interstitials. This process has been attested by specific color changes in the inair annealed films.

\section{The near-UV optical absorption in $\mathrm{AgNPs}_{\mathrm{TiO}}$ and hybrid $\mathrm{TiO}_{\mathrm{x}} / \mathrm{AgNPs}_{\mathrm{x}} / \mathrm{TiO}_{\mathrm{x}}$ films. The formation of the $\left[\mathrm{Ag}-\mathrm{TiO}_{\mathrm{x}}\right]$ complex.}

For the films containing AgNPs deposited on 1 or 3 supporting layers of $\mathrm{TiO}_{\mathrm{x}}$ (on sapphire) in comparison with the 40-layer pristine $\mathrm{TiO}_{\mathrm{x}}$ film as shown in Figure S6 and summarized in Table S1 (entries 1 and 3), the onset of the optical absorbance in the near-UV range appears to be bathochromically shifted despite the fact that the films with AgNPs contain only 1 or $3 \mathrm{TiO}_{\mathrm{x}}$ layers. Indeed, Figure S6 compares UVVIS spectra of the 18-nm thick pristine $\mathrm{TiO}_{\mathrm{x}}$ film with that of the bare small-AgNPs /1 $\left(\mathrm{TiO}_{\mathrm{x}}\right) /$ sapphire film (Table $\mathrm{S} 1$, entry 3 ) and displays the time evolution of the UVVIS spectrum of the later. First of all, a significant difference is evident in the nearUV spectral region between 200 and $400 \mathrm{~nm}$, which cannot be attributed to the AgSPR. At the spectral range below $330 \mathrm{~nm}$, the absorption coefficient, $\alpha$, appears to be discernibly larger for the silver-containing material in comparison with the pristine $\mathrm{TiO}_{\mathrm{x}}$ films suggesting that there are additional species absorbing in this region due to the presence of silver. The following can be suggested: a. the interband absorption of Ag metallic species which according to our measurements lies below $310 \mathrm{~nm}$; b. the absorption of silver cations, $\mathrm{Ag}^{+}$reported ${ }^{7,8}$ below $250 \mathrm{~nm}$; and c. the absorption of 
positively charged silver clusters, $\mathrm{Ag}_{\mathrm{n}}{ }^{\mathrm{m}+}$ reported ${ }^{6,7}$ between 270 and $350 \mathrm{~nm}$. With respect to the later two, it must be noted that these positively charged silver species are accommodated within the titania matrix as compensating ions coordinated with oxygen ions of the titania framework or in other forms.

Displayed in Figure S7, the difference spectra obtained during the evolution of this silver-containing film (Table S1, entry 3) under ambient atmospheric conditions reveal that, together with the rapid quenching of the AgNPs plasmon (the major negative-positive band), two positive bands at $312 \mathrm{~nm}$ and below $265 \mathrm{~nm}$ exhibit a steady growth. There is evidence ${ }^{6,8}$ to attribute the $312-\mathrm{nm}$ band to positivelycharged silver clusters, $\mathrm{Ag}_{\mathrm{n}}{ }^{\mathrm{m}+}$, and that below $265 \mathrm{~nm}$ to individual silver ions released by AgNPs in the course of the oxidative dissolution. These positivelycharged $\mathrm{Ag}$ species are most likely incorporated into the $\mathrm{TiO}_{\mathrm{x}}$ host forming an $[\mathrm{Ag}-$ $\mathrm{TiO}_{\mathrm{x}}$ ] complex despite a very large difference in the size of various silver species and Ti ions. The charge compensating position (the substitution of $\mathrm{H}^{+}$by the ion exchange reaction, $\mathrm{Ag}^{+}+\mathrm{HO}-\left[\mathrm{TiO}_{\mathrm{x}}\right] \rightarrow \mathrm{H}^{+}+\mathrm{Ag}-\mathrm{O}-\left[\mathrm{TiO}_{\mathrm{x}}\right]$, can be proposed. The absorption coefficient exhibits discernibly larger values for the AgNPs-containing films relative to the pristine $\mathrm{TiO}_{\mathrm{x}}$ matrix at the spectral range below $330 \mathrm{~nm}$ which can be attributed, on the one hand, to an additional contribution from the interband transition in metallic silver species and, on the other hand, to a contribution from the absorption of the $\left[\mathrm{Ag}-\mathrm{TiO}_{\mathrm{x}}\right]$ complex. It should be noted that while there is an obvious decay in the AgNPs plasmonic band the corresponding changes in the range below $330 \mathrm{~nm}$ are less pronounced (Figure S6 and Figure S7, the constant absorption coefficient at 260-270 nm, Figure S7) suggesting that the stability of some species contributing to the absorption in this range. On the other hand, the near-UV absorption tail for the AgNPs/(TiOx ) /sapphire film after 70 min under atmospheric conditions is found to continue down to $380-400 \mathrm{~nm}$ whereas the Urbach tail of the absorption of the pristine $\mathrm{TiO}_{\mathrm{x}}$ film is found stretched down only to $\sim 350 \mathrm{~nm}$ as follows from Figure S6 which again can be considered in supporting the notion of the $\left[\mathrm{Ag}-\mathrm{TiO}_{\mathrm{x}}\right]$ complex. Figure S8 reports values of direct and indirect optical gaps for this silver-containing film under ambient conditions which are found to be significantly smaller than respective values for the pristine $\mathrm{TiO}_{\mathrm{x}}$ matrix (see Table $\mathrm{S} 1$, entries 1 and 3) suggesting tentatively that the incorporation of different Ag species promotes a chemical modification within the amorphous $\mathrm{TiO}_{\mathrm{x}}$ structure. Furthermore, the change in the slope of respective optical curves with the exposure time it is also 
evident. This is in accord with the appearance of positive absorption bands in Figure S7. Therefore, it can be concluded on the basis of the present optical data that a metastable ensemble of as-deposited AgNPs upon the exposure to ambient atmospheric conditions evolves by the interaction with the $\mathrm{TiO}_{\mathrm{x}}$ substrate and atmospheric oxygen into the $\left[\mathrm{Ag}-\mathrm{TiO}_{\mathrm{x}}\right]$ complex in which positively charged silver species are coordinated with the oxygen ions of the titania framework. The titania framework is also affected by this process. Table S1 summarizes values of the onset of the optical absorption and optical gaps for different films prepared by the buffer assisted growth method and exposed to ambient atmospheric conditions. In particular, it can be observed that the hybrid film (entry 4) with two protective $\mathrm{TiO}_{\mathrm{x}}$ layers atop of AgNPs displays a distinctly low value of $3.74 \mathrm{eV}$ for the direct optical gap which remains practically constant during the exposure to atmospheric conditions which is in accord with a relatively slow decay of the Ag SPR evident from Figure 4B in the main manuscript for the dynamics of a similar hybrid film. In contrast to this stable performance in the near-UV range, the film (3) with bare AgNPs displays notable changes in the onset of the optical absorption and the direct optical gap together with a rapid quenching of the AgSPR. These observations imply again the correlation in the dynamics of the Ag plasmonic band and in the near-UV absorption under atmospheric conditions which can be interpreted in terms of the release of positively charged silver species by $\mathrm{AgNPs}$ and the formation of $\left[\mathrm{Ag}-\mathrm{TiO}_{\mathrm{x}}\right]$ complex. Questions associated with peculiarities of the oxidative dissolution of AgNPs and the dynamics of amorphous $\mathrm{TiO}_{\mathrm{x}}$ are under continuing studies in our laboratory.

\section{Analysis of the XPS Ag 3d profile and Ag photoelectron plasmon features.}

XPS measurements of samples containing AgNPs prepared by different deposition schemes are known to have difficulty in separating the metallic from the silver oxide peaks because they practically overlap. Nevertheless, XPS does reveal the presence of a plasmon resonance which self-manifests as plasmon satellites to $\mathrm{Ag}$ $3 \mathrm{~d} 5 / 2$ and $\mathrm{Ag} 3 \mathrm{~d} 3 / 2$ photoelectron peaks, known to arise from electron-energy-loss events of the photo-emitted electrons. Indeed, Figure S10 presents Ag 3d photoelectron spectra of two different samples containing ensembles of AgNPs. Sample A has been prepared by a direct deposition of $5 \AA$ of $\mathrm{Ag}$ atoms on the sapphire substrate (no buffer water layer has been employed). Sample B (TiOx /AgNPs / TiOx/ sapphire) has been prepared by 12 cycles of the deposition of $5 \AA \mathrm{Ag} / 50 \mathrm{ML}$ of $\mathrm{D}_{2} \mathrm{O}$ 
(about $60 \mathrm{~nm}$ diameter of the large particles, see Figure 3 in the main manuscript) on a $\mathrm{TiO}_{\mathrm{x}}$ layer grown by 40 cycles of $5 \AA \mathrm{Ti} / 50 \mathrm{ML}$ of $\mathrm{D}_{2} \mathrm{O}$ and then annealed in vacuum at $900 \mathrm{~K}$ for $45 \mathrm{~min}$. AgNPs have subsequently been protected by three additional titania layers of an identical composition of $5 \AA \mathrm{Ti} / 50 \mathrm{ML}$ of $\mathrm{D}_{2} \mathrm{O}$, followed by the immediate immersion in water after removal from the vacuum. It is evident that for both these samples one observes photoelectron plasmon (PEP) resonances located at $3.1 \mathrm{eV}$ (Figure S10 A) and 3.0 eV (Figure S10 B), from the respective photoelectron Ag 3d peaks. Our additional XPS measurements (not presented here) show that the spectral position of these PEP resonances displays a clear dependence on the dielectric characteristics of an immediate environment of AgNPs which is at least in a partial accord with the assignment of PEPs to the surface resonance.

XPS measurements reveal PEPs also for those samples which display no optical plasmon activity despite the presence of AgNPs in the metallic state. Indeed, while Figure S7 A clearly displays the PEP peaks, UV-VIS measurements on this sample have shown that the optical plasmon resonance has been quenched after 30 minutes exposure to an ambient atmosphere prior to placing the sample in the XPS instrument. On the other hand, sample B with a significantly larger number of AgNPs of a larger average diameter and the titania protecting layers shows both a strong and stable optical plasmon and the PEP feature. There is also a noticeable difference in the frequency position of the optical surface plasmon and PEP resonances as can be inferred from the comparison of Figure 3B, which displays the optical surface plasmon feature at $530 \mathrm{~nm}$, or $2.4 \mathrm{eV}$, and Figure S7 B, which displays the PEP feature at $3.0 \mathrm{eV}$, for two comparable samples. It is possible that the observed difference reflects a variation in electrostatic charging, or in the electrochemical equilibrium of AgNPs in the ambient atmosphere and in the vacuum. 


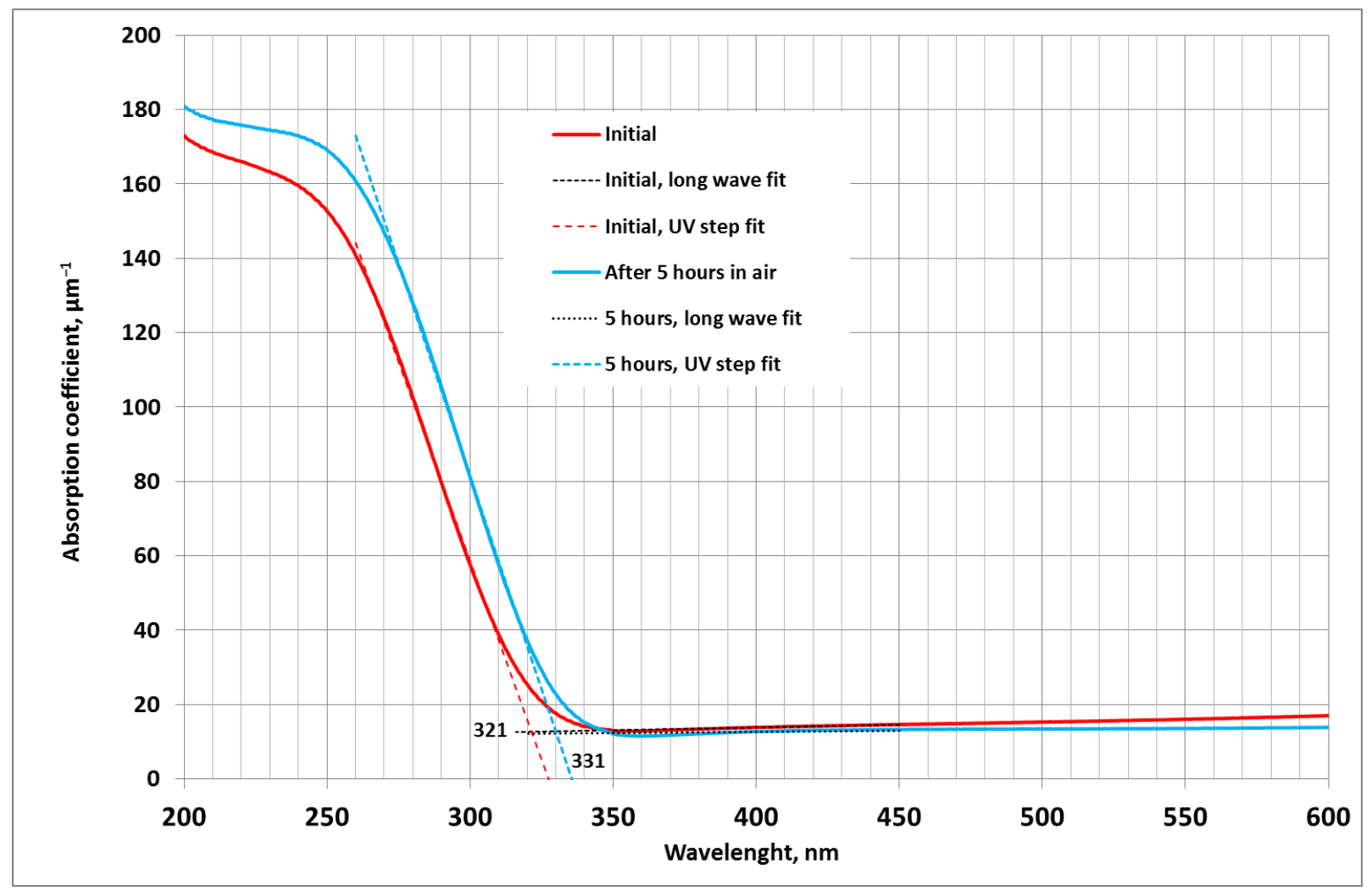

\section{Figure S1}

The evolution of the optical absorption of an amorphous 18-nm-thick TiOx film prepared by 40 cycles of the RLAD procedure upon exposure to an ambient atmosphere. The red absorption curve is for the film immediately after the exposure to an atmospheric environment; the blue curve is for the same film after 5 hours into the atmospheric exposure. The onset of the optical absorption in the near-UV region are found at 321 and $331 \mathrm{~nm}$. 


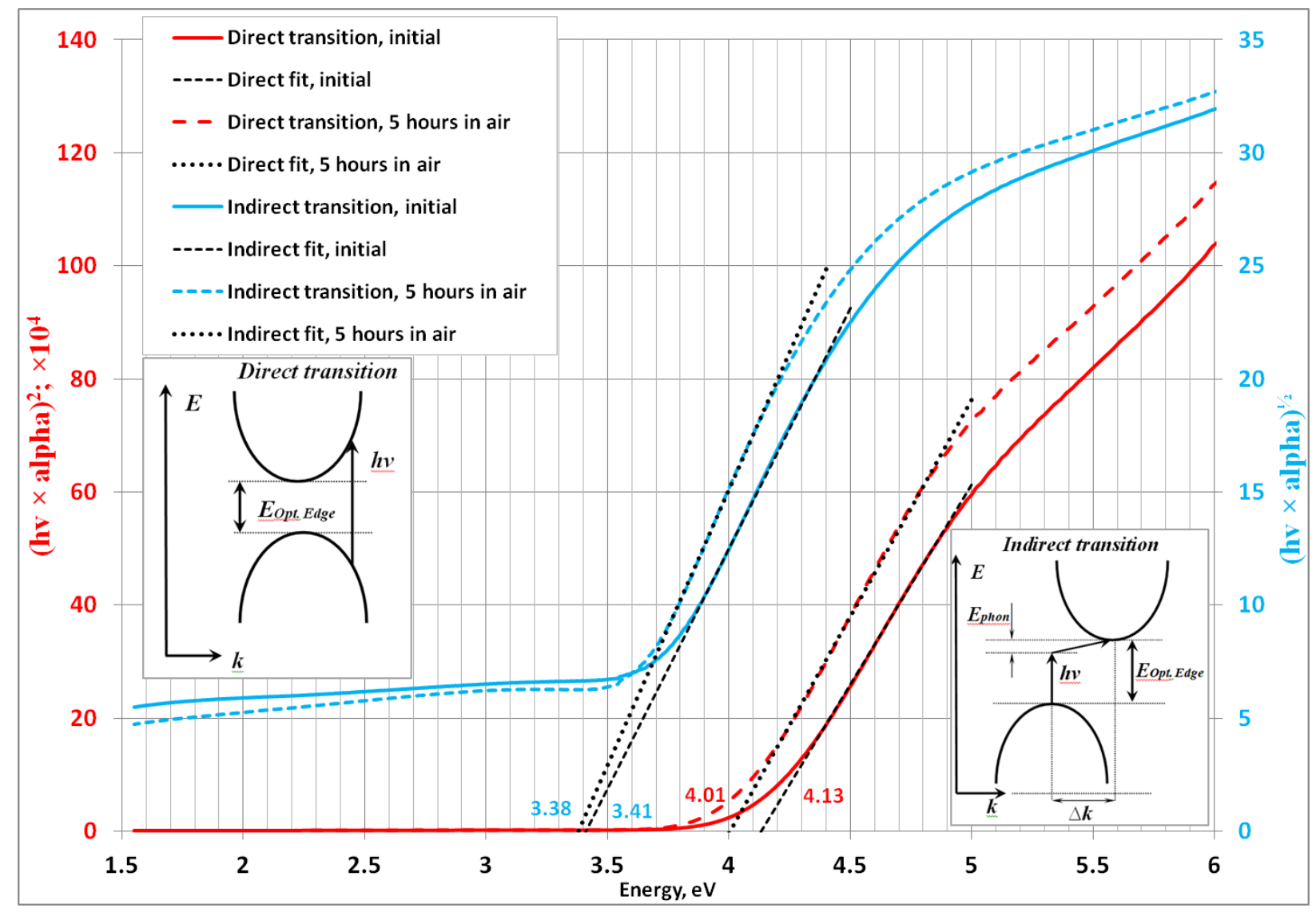

\section{Figure S2}

Optical edges for an amorphous TiOx film prepared by 40 cycles of the RLAG at the initial moment and after 5 hours of the exposure at ambient atmospheric conditions. For the direct and indirect transitions the ordinate scale is $(h v \times \alpha)^{2}$ and $(h v \times \alpha)^{1 / 2}$, respectively, according to Eqs. (2) and (3) (see above in the supporting information text), $\alpha$ is the absorption coefficient of the $\mathrm{TiO}_{\mathrm{x}}$ film with the thickness of $18 \mathrm{~nm}$. The cartoons describe the direct and indirect transitions in the $E, k$ coordinate space where $k$ stands for the momentum coordinates. 


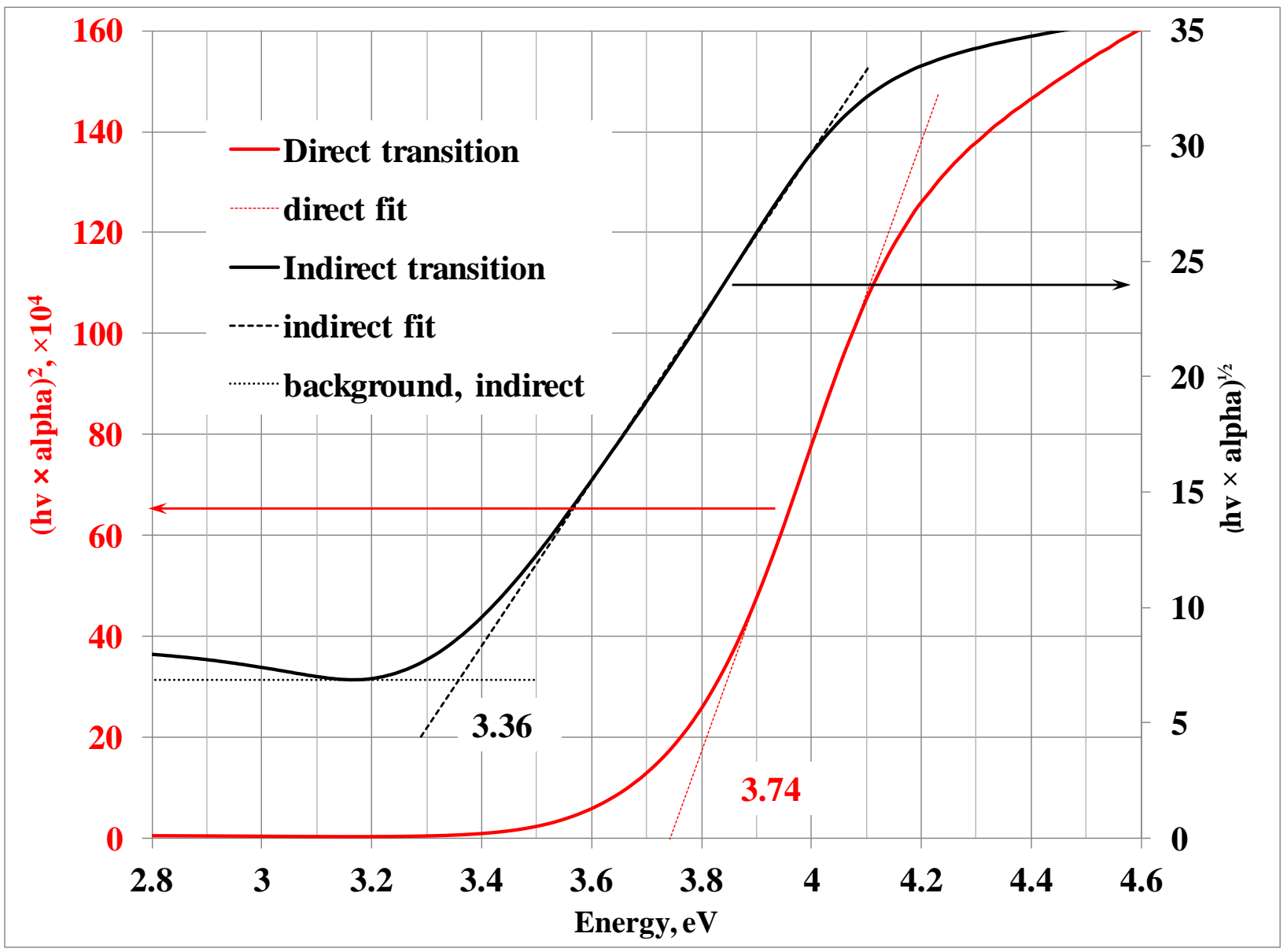

\section{Figure S3}

Optical edges for the nanocrystalline rutile film prepared by 40 cycles of the RLAG and annealed at $1000 \mathrm{~K}$ in air for 1 hour. The optical edges are obtained in the direct and indirect coordinates, respectively, in spite of the direct band gap in the macroscopically large rutile samples. The Scherrer crystalline size is $10 \mathrm{~nm}$. 


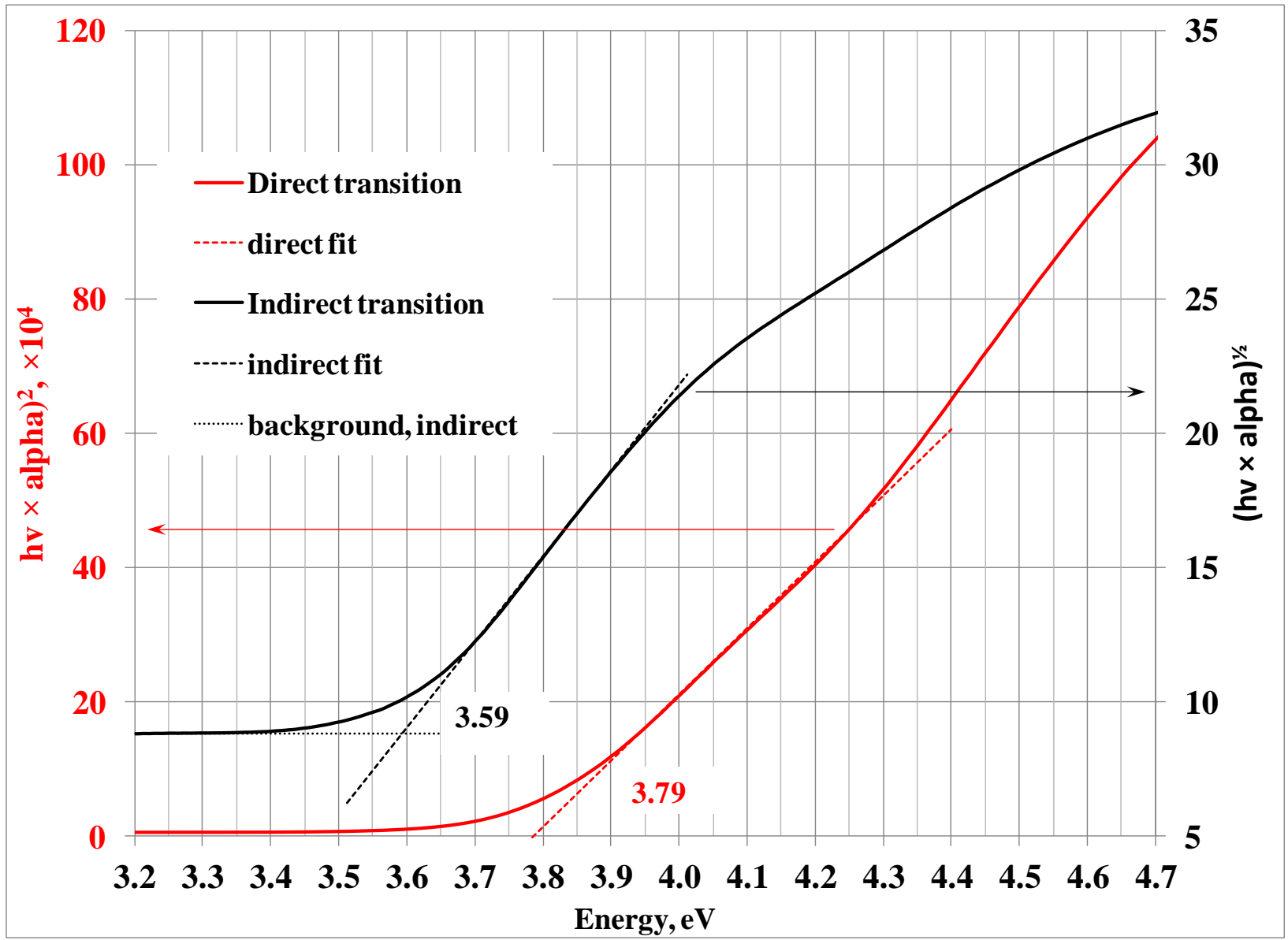

\section{Figure S4}

Optical edges for the nanocrystalline anatase film obtained from as-grown by 40 cycles RLAG amorphous titania film via a complex annealing procedure. The Scherrer crystalline size is $50 \mathrm{~nm}$. 


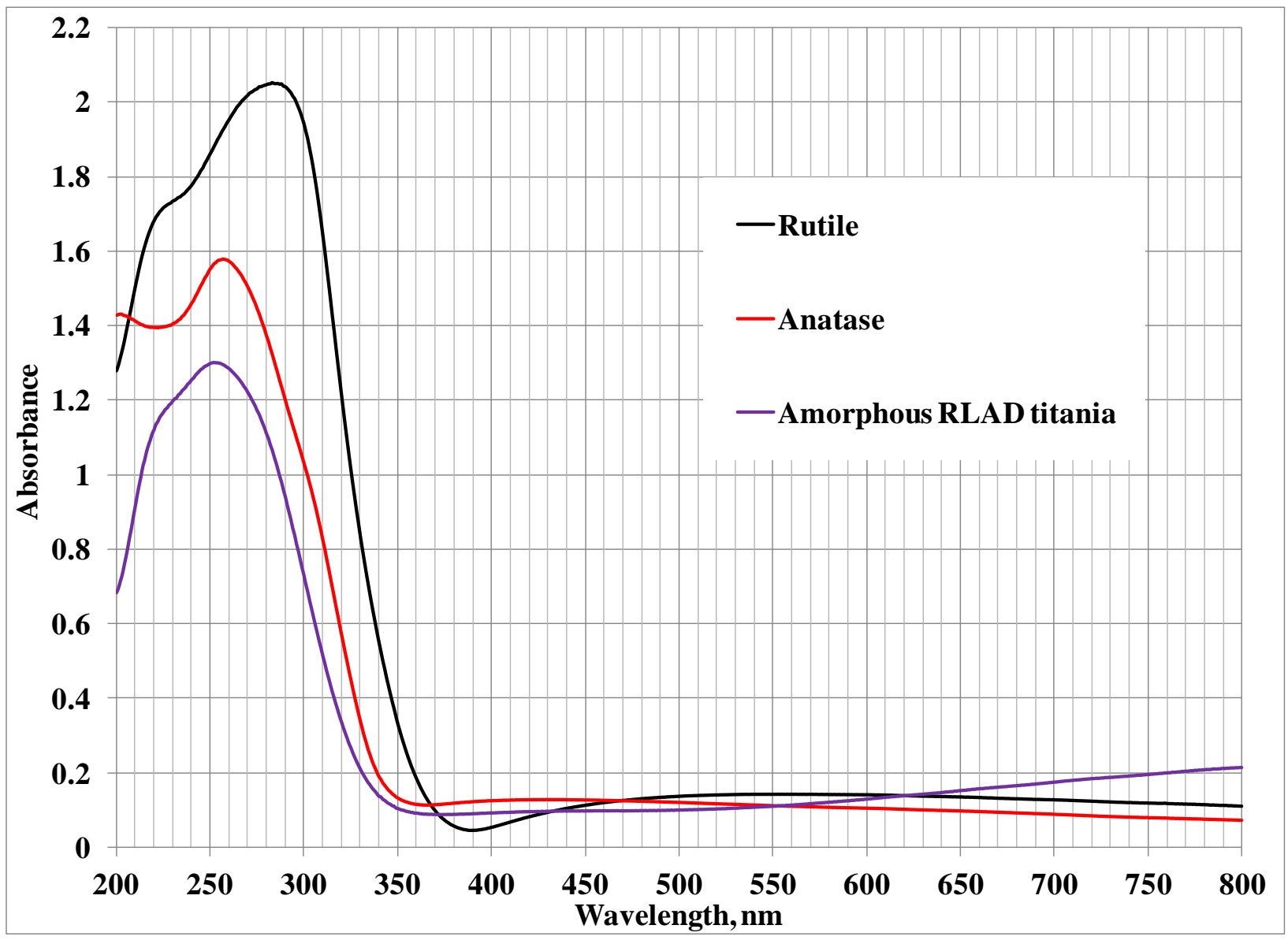

\section{Figure S5}

UV-vis spectra of the amorphous RLAD titania film obtained by 40 cycles and nanocrystalline rutile and anatase films obtained after different annealing procedures in atmospheric conditions. The spectrum of the amorphous film after in-vacuum annealing at $775 \mathrm{~K}$ is indistinguishable from that of the as-grown film. 


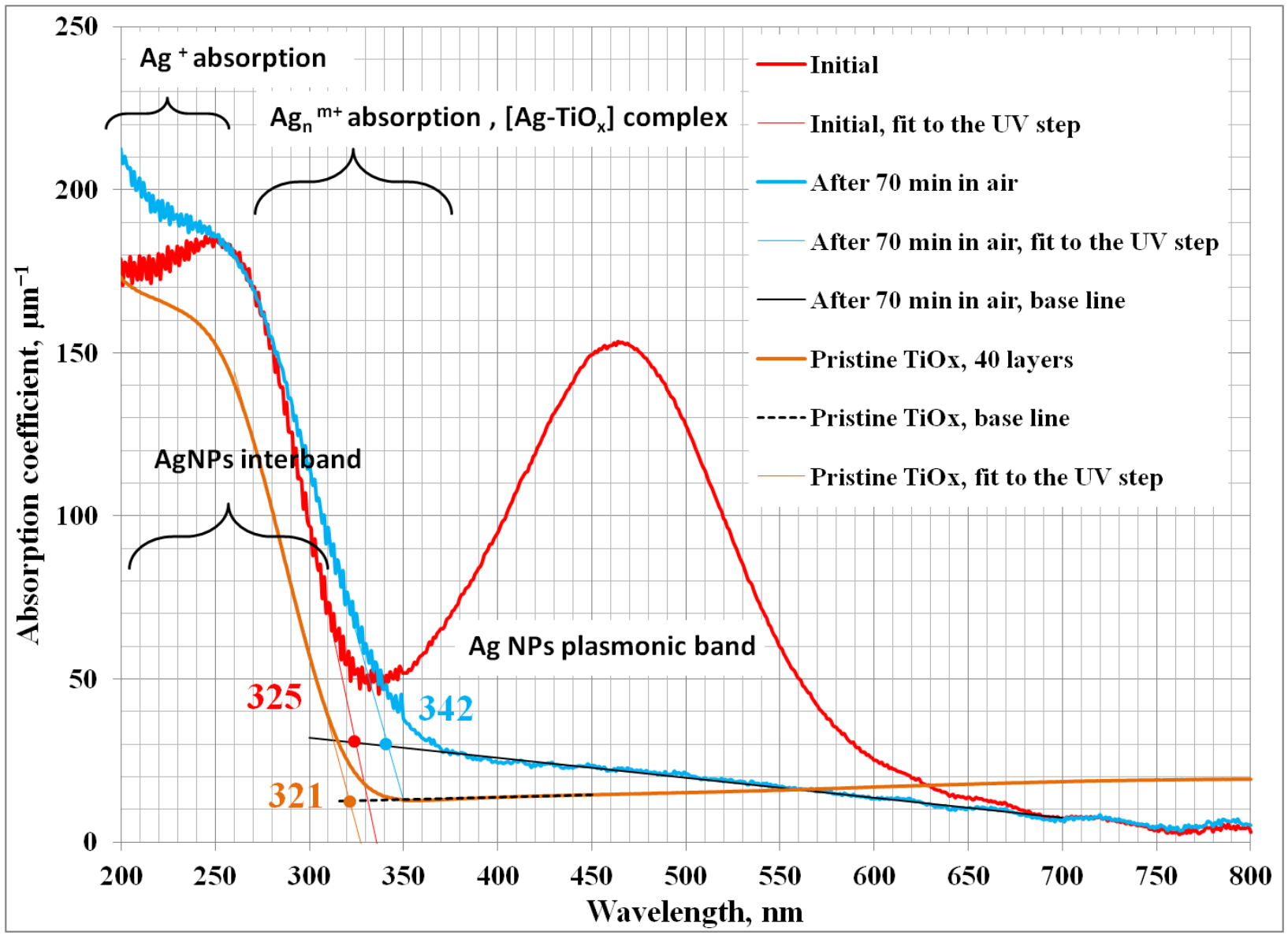

\section{Figure S6}

The evolution of the optical absorption of the bare-AgNPs/TiOx/sapphire film upon exposure to an ambient atmosphere. The thickness of $\mathrm{TiO}_{\mathrm{x}}$ matrix is estimated at 4.5 $\AA$. The red absorption curve is for the film at the initial moment of the exposure; the blue curve is for the film after $70 \mathrm{~min}$ exposure to ambient conditions. The onset of the optical absorption is found at $325 \mathrm{~nm}$ and $342 \mathrm{~nm}$, respectively. Absorption regions for $\mathrm{Ag}^{+}$cations, $\mathrm{Ag}_{\mathrm{n}}{ }^{\mathrm{m}+}$ positively charged clusters or $\left[\mathrm{Ag}-\mathrm{TiO}_{\mathrm{x}}\right]-$ complex, the AgNPs interband and the Ag SPR are labeled. The spectrum of the pristine 18-nm thick $\mathrm{TiO}_{\mathrm{x}}$ film (amber line) at the initial moment of the exposure is added as a reference for comparison. 


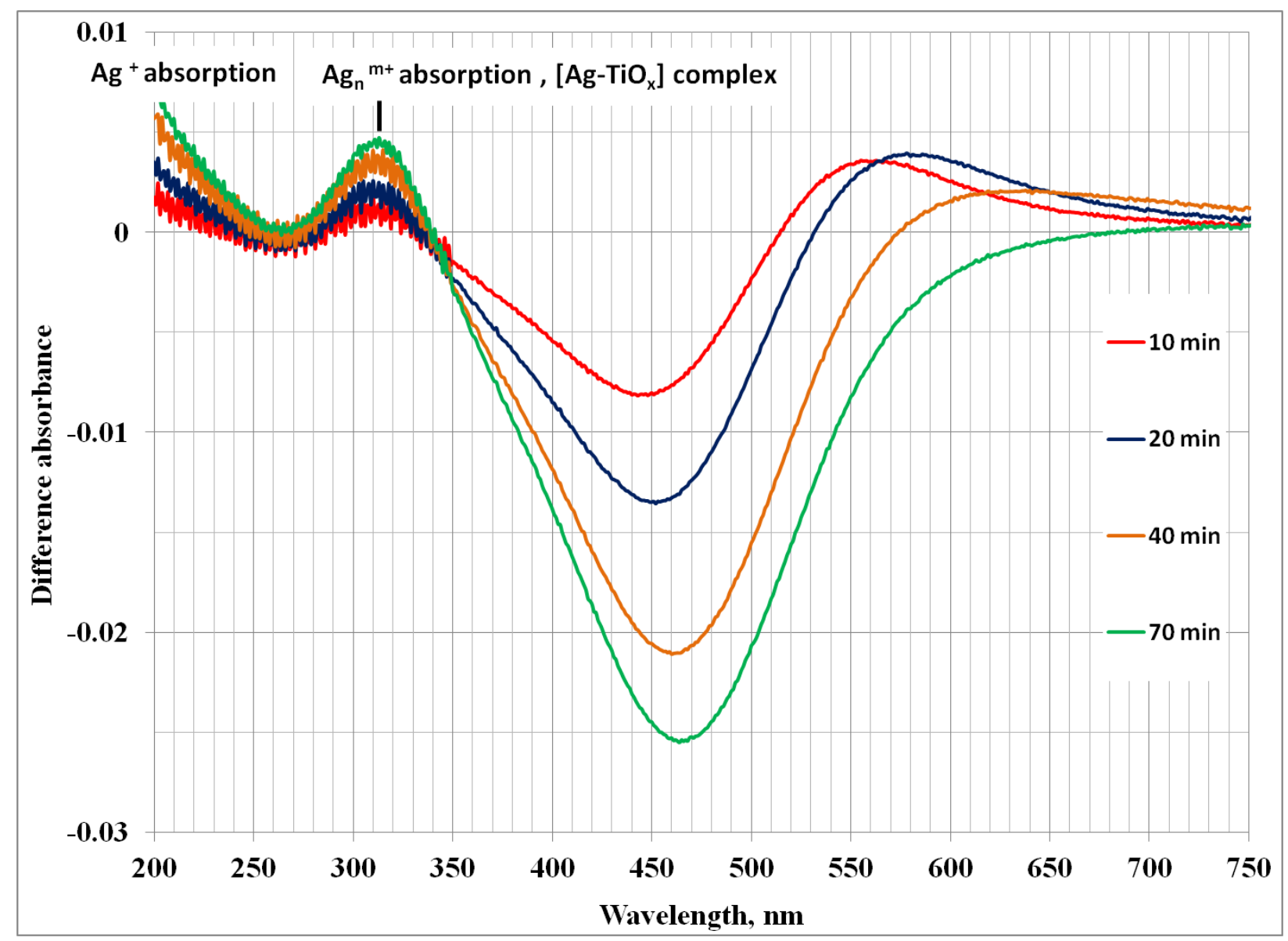

\section{Figure S7}

Difference absorption UV-vis spectra for the bare AgNPs/TiO $/$ /sapphire film obtained at 10, 20, 40 and 70 min exposure to an ambient atmosphere, i.e. Spec. (10 min) - Spec. (1 min); Spec. (20 min) - Spec. (1 min); Spec. (40 min $)$ - Spec. (1 min); and Spec. (70 $\mathrm{min})$ - Spec. (1 min). Two positive bands, one below $260 \mathrm{~nm}$ and the second at $312 \mathrm{~nm}$, are attributed to the emergence of $\mathrm{Ag}^{+}$ions and $\mathrm{Ag}_{\mathrm{n}}{ }^{\mathrm{m}+}$ clusters or $\left[\mathrm{Ag}-\mathrm{TiO}_{\mathrm{x}}\right]$ complex, respectively.

The major negative-positive band between $350-750 \mathrm{~nm}$ is associated with the decay of the Ag SPR band. 


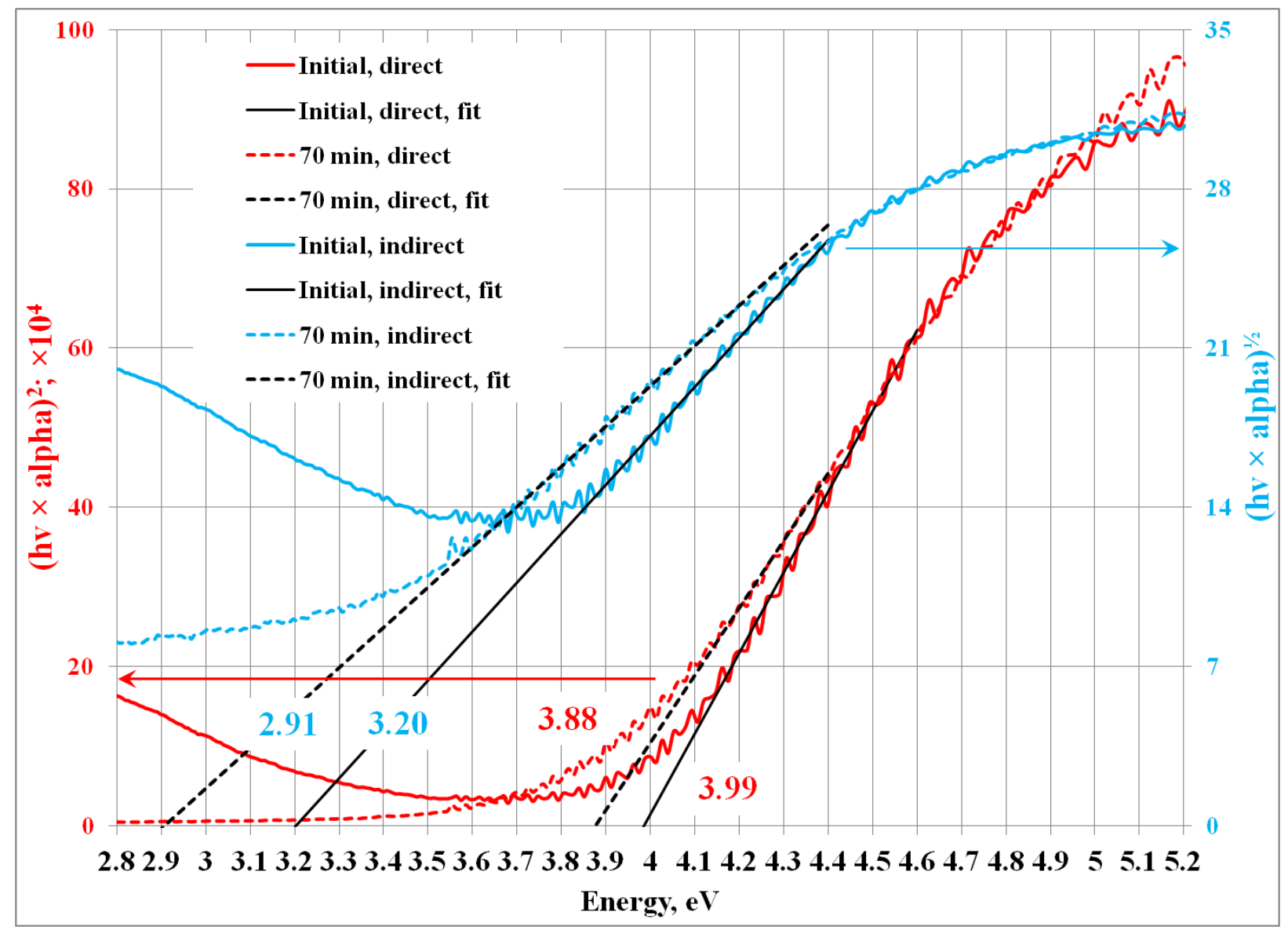

\section{Figure S8}

Optical edges for a film of bare-AgNPs/ $1\left(\mathrm{TiO}_{\mathrm{x}}\right) /$ sapphire at the initial moment and after 70 min into the exposure to an ambient atmosphere. The thickness of $\mathrm{TiO}_{\mathrm{x}}$ matrix is estimated at $4.5 \AA$. For the direct and indirect transitions the ordinate scales are $(h v \times \alpha)^{2}$ and $(h v \times \alpha)^{1 / 2}$, respectively, according to Eqs. S(2) and S(3). 


\begin{tabular}{|c|c|c|c|c|}
\hline & \multirow[t]{2}{*}{ Film composition } & \multirow{2}{*}{$\begin{array}{l}\text { № of } \\
\mathrm{TiO}_{\mathrm{x}} \\
\text { layers }\end{array}$} & \multicolumn{2}{|c|}{$\begin{array}{l}\text { Onset of the optical absorption, nm (eV); } \\
\text { Optical edges (Direct / Indirect) (eV) }\end{array}$} \\
\hline & & & Initial & Atm. exposure, $\min$ \\
\hline 1 & $40\left(\mathrm{TiO}_{\mathrm{x}}\right) / \mathrm{Al}_{2} \mathrm{O}_{3}$ & 40 & $\begin{array}{l}321(3.86) ; \\
4.13 / 3.41\end{array}$ & $\begin{array}{l}330(3.76)_{300 \mathrm{~min}} ; \\
4.01 / 3.38_{300 \mathrm{~min}}\end{array}$ \\
\hline 2 & $3\left(\mathrm{TiO}_{\mathrm{x}}\right) / \mathrm{Al}_{2} \mathrm{O}_{3}$ & 3 & $\begin{array}{c}337(3.68) \\
3.72 \mathrm{Dir}\end{array}$ & $\begin{array}{c}336(3.69)_{24} \text { hours } \\
3.72 \text { Dir, } 24 \text { hours }\end{array}$ \\
\hline 3 & Small-AgNPs/1 $\left(\mathrm{TiO}_{\mathrm{x}}\right) / \mathrm{Al}_{2} \mathrm{O}_{3}$ & 1 & $\begin{array}{l}325(3.82) \\
3.99 / 3.20\end{array}$ & $\begin{array}{l}342(3.63) 90 \mathrm{~min} \\
3.88 / 2.9190 \mathrm{~min}\end{array}$ \\
\hline 4 & $2\left(\mathrm{TiO}_{\mathrm{x}}\right) / \mathrm{small}-\mathrm{AgNPs} / 1\left(\mathrm{TiO}_{\mathrm{x}}\right) / \mathrm{Al}_{2} \mathrm{O}_{3}$ & 3 & $\begin{array}{c}326(3.80) \\
3.74 \text { Dir }\end{array}$ & $\begin{array}{l}329(3.77)_{190 \min } \\
3.73_{\mathrm{Dir}, 90 \mathrm{~min}}\end{array}$ \\
\hline 5 & $\begin{array}{l}\text { Nanocrystalline rutile film, } \\
\text { the Scherrer crystalline size } 10 \mathrm{~nm}\end{array}$ & 40 & \multicolumn{2}{|c|}{$3.74 / 3.36$ relative to the background } \\
\hline 6 & $\begin{array}{l}\text { Nanocrystalline anatase film, } \\
\text { the Scherrer crystalline size } 50 \mathrm{~nm}\end{array}$ & 40 & \multicolumn{2}{|c|}{$3.79 / 3.59$ relative to the background } \\
\hline
\end{tabular}

Table S1

The onset of the UV-optical absorption and optical gaps for different films prepared by the buffer layer assisted growth methods, exposure to ambient atmospheric conditions and crystallized to rutile and anatase phases by different annealing procedures. The estimated thickness of a single $\mathrm{TiO}_{\mathrm{x}}$ layer is $4.5 \AA$. The measured thickness of the amorphous titania film prepared by 40 cycles of RLAD is $18 \mathrm{~nm}$. 

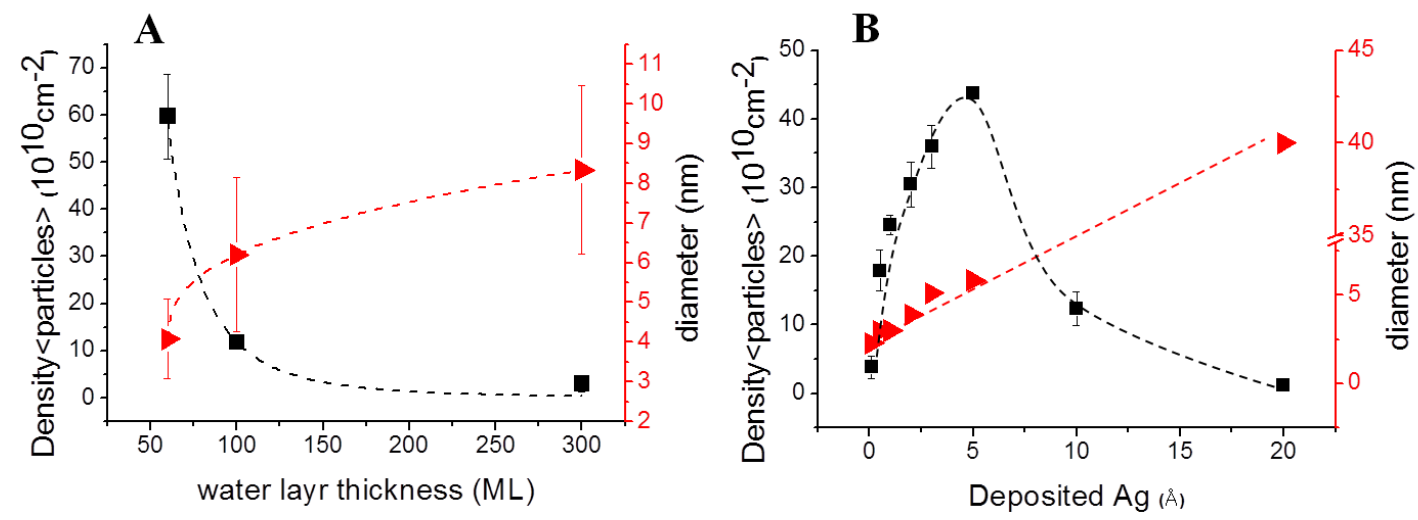

\section{Figure S9}

A. Dependence of the size (in red) and the density (in black) of AgNPs on the water layer thickness. B. Dependence of the size (in red) and density (in black) of AgNPs on the amount of deposited silver. 

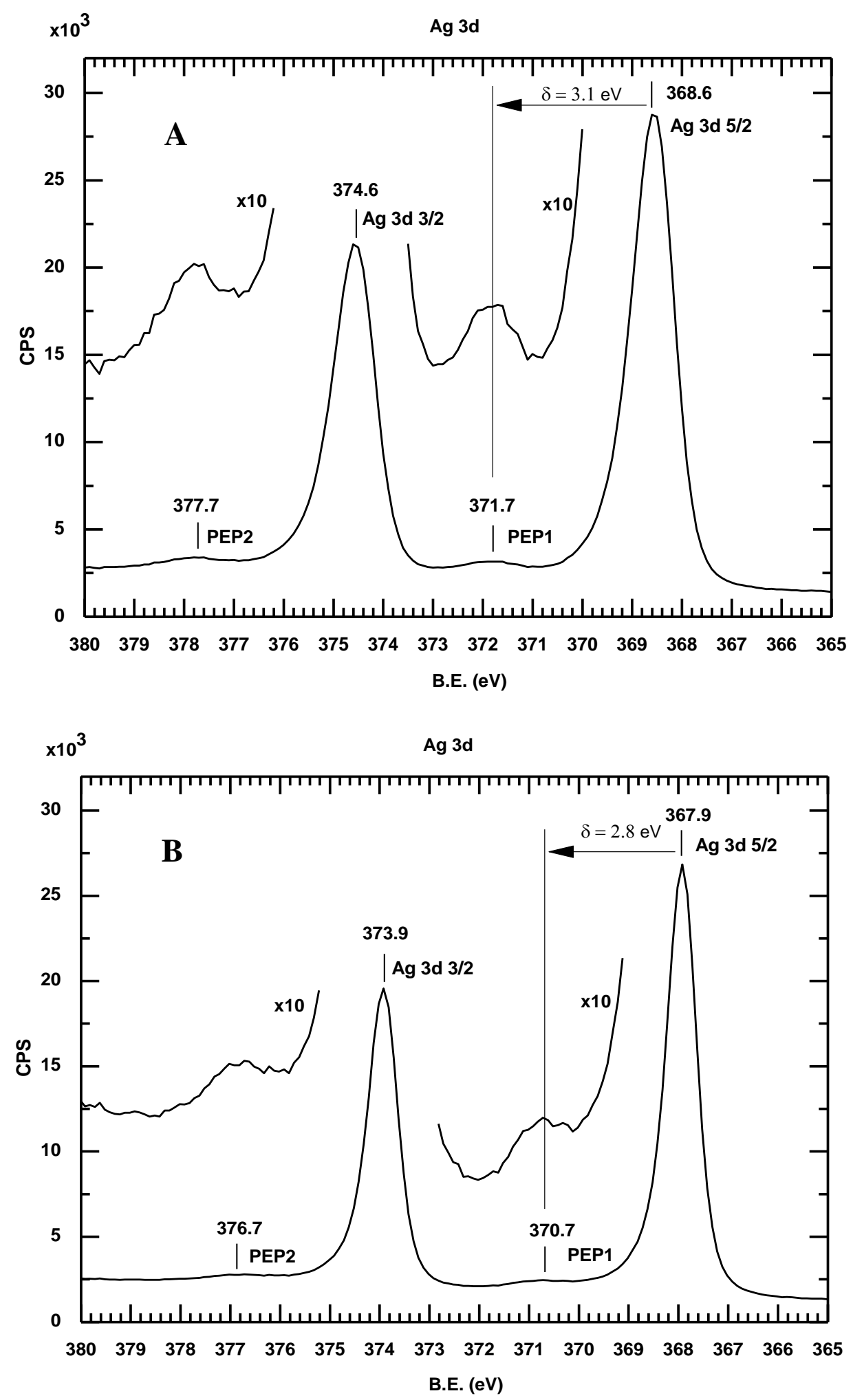

\section{Figure S10}

A. XPS measurements of bare-AgNPs (4nm in diameter) showing that PEP peaks at $371 \mathrm{eV}$ appears in the absence of the optical plasmon. B. The PEP of titania-protected AgNPs sample exhibiting relatively stable optical SPR absorption. The location of PEP is identical to that in sample A. 


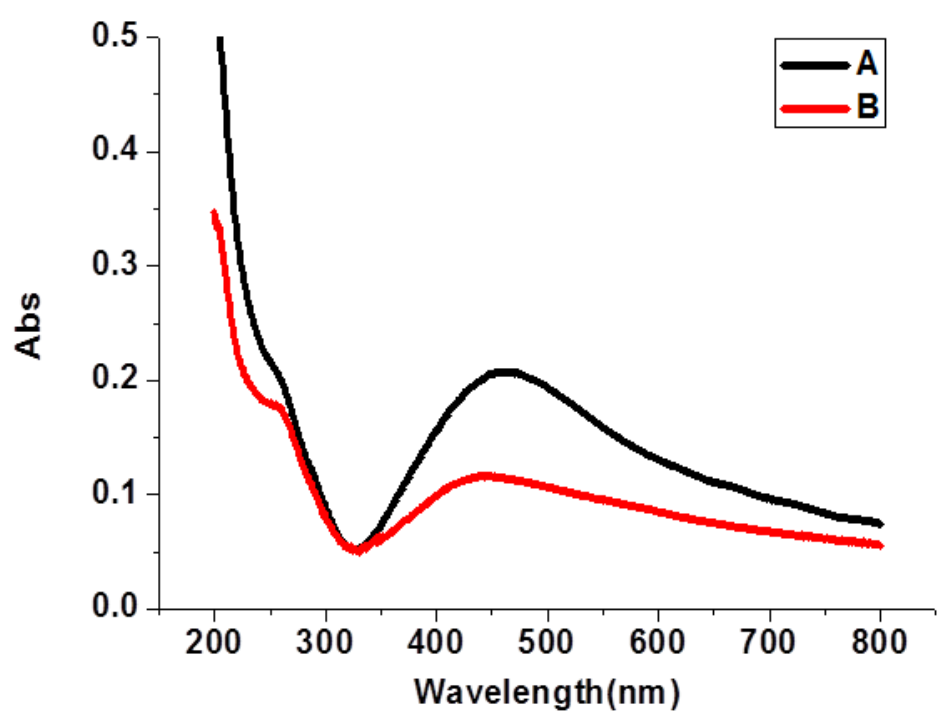

\section{Figure S11}

The optical properties of larger AgNPs ( $80 \pm 20 \mathrm{~nm}$ in diameter) were examined using a spectrophotometer (Varian Cary 5000) equipped with an integrating sphere. The two spectra are: A. Total extinction (absorption + scattering) (in black) with the sample located at the entrance to the sphere. B. Pure absorption signal (in red) obtained via the integrating sphere apparatus attached to the CARY 5000 spectrophotometer, with the sample located at the center of the sphere. The pure absorption is about $45 \%$ of the total extinction signal, which means that remaining $55 \%$ is due to light scattering from the relatively large AgNPs. 


\section{References:}

(1) T. G. Moss, G. J. Burrell, E. Ellis, Semiconductors opto-electronics, Butterworth, London, 1973.

(2) X. K. Zbao, J. H. Fendler, Size quantlzatlon in semiconductor particulate films. J. Phys. Chem. 1991, 95, 3716-3723.

(3) J. Tauc, Absorption edge and internal electric fields in amorphous semiconductors. Mat. Res. Bull. 1970, 5, 721-730.

(4) E. Joselevich, I. Willner, Photosensitization of quantum-size $\mathrm{TiO}_{2}$ particles in water-in-oil microemulsions. J. Phys. Chem. 1994, 98, 7628-7635.

(5) N. Serpone, D. Lawless, R. Khairutdinov, Size effects on the photophysical properties of colloidal anatase $\mathrm{TiO}_{2}$ particles: size quantization or direct transitions in this indirect semiconductor? J. Phys. Chem. 1995, 99, 16646-16654.

(6) M. Radecka, K. Zakrzewska, H. Czternastek, T. Stapiriski, The influence of thermal annealing on the structural, electrical and optical properties of $\mathrm{TiO}_{2-\mathrm{x}}$ thin films. App. Sur. Sci. 1993, 65/66, 227-234.

(7) A. Henglein, Physicochemical properties of small metal particles in solution: "microelectrode" reactions, chemisorption, composite metal particles, and the atomto-metal transition. J. Phys. Chem. 1993, 97, 5457-5471.

(8) P. Sazama , L. Capek , H. Drobná, Z. Sobalík , J. Dedecek , K. Arve , B.

Wichterlová, Enhancement of decane-SCR-NO ${ }_{x}$ over Ag/alumina by hydrogen.

Reaction kinetics and in situ FTIR and UV-vis study. J. Catal. 2005, 232, 302-317. 